\title{
CAPÍTULO 40: DESENVOLVIMENTO E AVALIAÇ̃̃O DE UM CARRINHO AUTÔNOMO PARA A PROSPECÇÃO DE VARIÁVEIS PRODUTIVAS NO CULTIVO DE MORANGO DE PEQUENOS AGRICULTORES DA BACIA DE YALI (REGIÃO METROPOLITANA)
}

\section{CAPÍTULO 40: DESARROLLO Y EVALUACIÓN DE UN CARRO AUTÓNOMO PARA PROSPECCIÓN DE VARIABLES PRODUCTIVAS EN CULTIVO DE FRUTILLAS DE PEQUEÑOS AGRICULTORES DE LA CUENCA DEL YALI (REGIÓN METROPOLITANA)}

\section{CHAPTER 40: DEVELOPMENT AND EVALUATION OF AN AUTONOMOUS TROLLEY FOR PROSPECTING PRODUCTIVE VARIABLES IN STRAWBERRY CULTIVATION OF SMALL FARMERS IN THE YALI BASIN (METROPOLITAN REGION)}

Ninrod Mena Zumarán ${ }^{1}$; Andrés Honeyman²; Rubén Moreno; Cecilia Gutiérrez ${ }^{4}$

DOI: https://doi.org/10.31692/978-65-88970-07-2.353-357

\section{INTRODUCCIÓN}

En el contexto del Acuerdo Voluntario para la Gestión de Cuenca (AVGC) impulsado por el Ministerio del Medio ambiente y la Agencia de sustentabilidad y Cambio Climático, estudiantes de INACAP, del área de Informática y Telecomunicaciones, de la carrera de Telecomunicaciones, Conectividad y Redes, en conjunto con las áreas de Agroindustria y Medio Ambiente y Mecánica, se encuentran desarrollando un prototipo a escala real de un vehículo automatizado que permitirá realizar pruebas en terreno para verificar el vigor vegetativo de plantaciones de frutillas de agricultores localizados en la Comuna de San Pedro de la Provincia de Melipilla, Región Metropolitana, Santiago, Chile. Específicamente el trabajo se desarrolla en la Cuenca de El Yali, sector de la provincia históricamente reconocido por la producción de esta especie y actualmente en tramitación del Sello de origen de las Frutillas de San Pedro, sector muy afectado por la mega sequía de los últimos 10 años que hace cada vez más difícil la producción de esta especie y la subsistencia de sus agricultores. El objetivo del carro es utilizar tecnologías de la información y comunicaciones para el envío de datos y posterior análisis, es importante la selección de los softwares para el control de los equipos de red que serán utilizados, y pensando en prevenir posibles ataques de malware y

\footnotetext{
1 Telecomunicaciones Conectividad y Redes, INACAP, ninrod.mena@inacapmail.cl

2 Ingeniería Agrícola, INACAP, andres.honeyman@inacapmail.cl

3 Mecánica, INACAP, INACAP, ruben.romero@inacapmail.cl

4 Vicerrectoría académica, INACAP, cguti@inacap.cl
} 
otros de este tipo se deberán establecer políticas de seguridad y monitoreo remoto.

El uso de cámara multiespectral sobre carro autónomo se utiliza para la determinación del índice de vigor NDVI, generando mapas de vigor sitio especifico, que, con registros del manejo de fertilización y riego, permitirán implementar una estrategia sustentable para los cultivos. En su primera etapa se espera contar con este carro para poder implementar y montar los equipos necesarios que permitan obtener muestras de las plantas de frutillas, es decir, el sensor NDVI, un GPS para geolocalización, un equipo móvil y antenas que permitan el enlace con un servidor de almacenamiento de datos.

La metodología utilizada es el aprendizaje a través de metodologías activas (Nel, 2017; INACAP, 2018), cuya estrategia metodológica es el ABPro y se inserta en una asignatura de la Carrera Telecomunicaciones, Conectividad y Redes (Aksela and Haatainen, 2018).

Los docentes que participan fueron seleccionados según las asignaturas que dictan y las necesidades del proyecto. Los estudiantes, de cuarto semestre, que participan lo hacen de manera voluntaria y eligen un proyecto en el contexto de su asignatura de Taller de Integración I. Los estudiantes son invitados por cada docente usando 3 criterios: i) por invitación del respectivo docente, ii) por interés personal del estudiante y/o iii) por su progresión académica.

\section{INFORME DE EXPERIENCIA}

El Objetivo general del Proyecto es desarrollar y evaluar un vehículo autónomo para apoyar la toma de decisiones asociadas a variables productivas en cultivo de frutillas de pequeños y medianos agricultores. Los objetivos específicos son: 1) Desarrollar un vehículo autónomo capaz de transportar los equipos de prospección de parámetros productivos a través del cultivo de frutilla, en forma rápida y segura, 2) Desarrollar una estructura de telecomunicación que permita, tanto el control del vehículo como la transmisión de los datos recolectados, 3) Evaluar el desempeño del vehículo y su capacidad de adaptación a las diferentes realidades de los agricultores beneficiarios y 4) Transformar los datos recolectados en información útil para la toma de decisiones por parte de los agricultores beneficiarios, mediante agricultura de precisión.

Fases desarrolladas en el Proyecto. Objetivo específico 1: "Desarrollar un vehículo autónomo capaz de transportar los equipos de prospección de parámetros productivos a través del cultivo de frutilla, en forma rápida y segura.” Al respecto este objetivo está parcialmente 
logrado, el carro se ha diseñado (v3) y se tiene un modelo a escala que requiere el cableado eléctrico y la instalación de los motores. Respecto de los motores se realizó la programación a escala en motores paso a paso para un carro de juguete y se controló a través de una placa Arduino. Objetivo específico 2: "Desarrollar una estructura de telecomunicación que permita, tanto el control del vehículo como la transmisión de los datos recolectados." Propuesta desarrollada con configuración, lograda y probada, se ve en la figura 02 y la configuración utilizada en prototipo a escala en la figura 03. Para ello es necesario el uso de programación de Arduino y hoy se utilizarán facilidades otorgadas por la nube de AWS para la configuración de bases de datos que alojen la información recopilada, además se agregará seguridad a las conexiones remotas de modo prevenir modificaciones accidentales o intensionales en los datos almacenados.

Figura 01: Cultivo de frutilla,

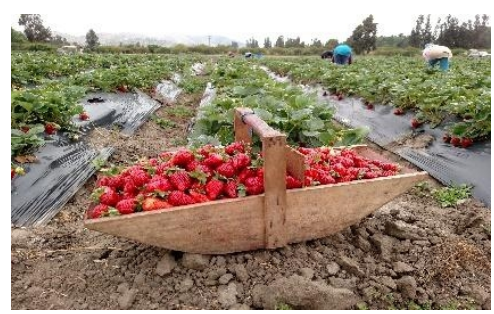

Fuente: Propia (2019).

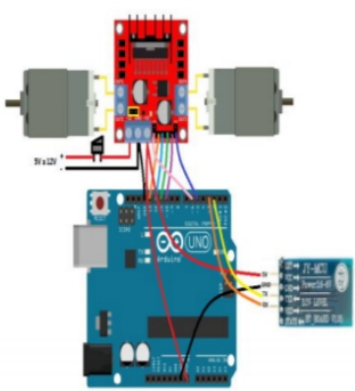

Figura 02: Diseño general de Carro autónomo y prototipo escala

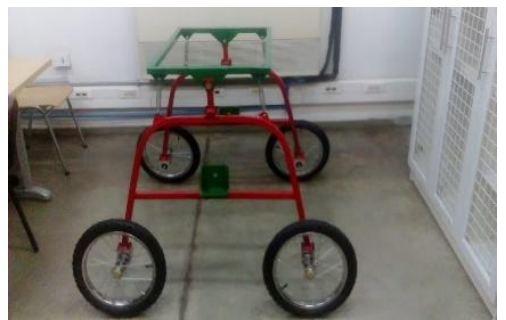

Fuente: Propia (2019).

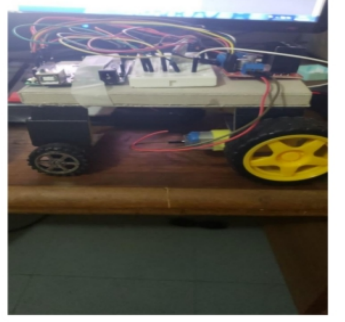

Fuente: Propia (2019).
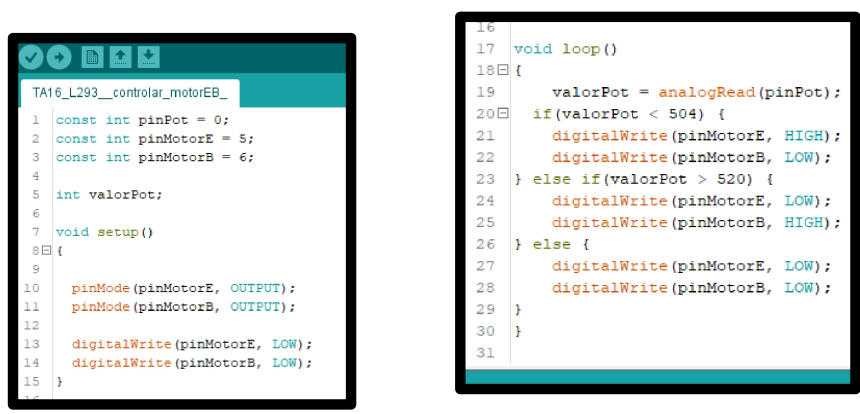

Figura 03: Topología de conexión Arduino como propuesta de control del carro.

Fuente: Propia (2019).

Las pruebas del carro a escala están funcionando según lo programado, falta avanzar a los objetivos específicos 3 y 4, que por problemas de la pandemia COVID19 y la imposibilidad de entrar a los campus para trabajar en el montaje de los motores, no ha sido posible llevar a cabo en este período de tiempo. Se requiere contar con el carro y sus motores ya instalados, para poder realizar:

a) Evaluación del desempeño del vehículo y su capacidad de adaptación a las diferentes realidades de los agricultores beneficiarios. 
b) Transformar los datos recolectados en información útil para la toma de decisiones por parte de los agricultores beneficiarios, mediante agricultura de precisión.

Esperamos durante la P2020 poder llevar a terreno y realizar pruebas de campo y verificar su funcionamiento a escala real, esto permitirá ajustar variables y otros antes de entregar el producto terminado.

\section{CONCLUSIONES}

Esta experiencia de aprendizaje en vínculo con las necesidades de los agricultores ha sido una nueva experiencia para los estudiantes de las carreras de Conectividad y Redes y especial desafío ya que ellos normalmente no se relacionan con el medio rural y los cultivos. A la fecha han participado en este carro dos grupos de estudiantes de cuarto semestre del área de Telecomunicaciones, Conectividad y Redes, cada uno de tres personas; dos alumnos del área de mecánica, dos de electricidad y cuatro de Ingeniería agrícola, de manera multidisciplinaria han compartido interés y motivación, aplicando sus conocimientos y competencias en el desarrollo de sus proyectos de taller. La metodología ABPro a demostrado ser la adecuada para el abordaje de los objetivos plateados.

Los alumnos del área de Telecomunicaciones, Conectividad y Redes em conjunto con sus compañeros de Ingeniería Agrícola, Mecánica y Electricidad aceptan el desafío de aplicar sus conocimientos teóricos en un caso real, lo que los lleva a buscar las mejores soluciones em cada una de sus áreas.

En particular se observa que los grupos del área de Telecomunicaciones aceptan el proyecto y basados em el desarrollo del grupo anterior buscan mejorar las prestaciones del carro y proponen el uso de un equipo notebook que permita la configuración del software SMS Mobile para la recolección de datos (este se utiliza en conjunto con sensor Ag Leader OptRx modelo ACS430) y conexión a internet. Haciendo uso de tecnología 3G se propone la instalación de una antena Yagi Air802 para realizar un enlace de respaldo, sin embargo, esto último queda a la espera de tener el carro funcional y poder realizar las primeras pruebas para comenzar con el ciclo de Deming.

\section{REFERENCIAS}

[356] 
Nel, L. (2017). Students as collaborators in creating meaningful learning experiences in technology -enhanced classrooms: an engaged scholarship approach. Available in:

https://onlinelibrary.wiley.com/doi/pdf/10.1111/bjet.12549

INACAP. (2018). Congreso Internacional Active Learning: Aprendizaje Activo en Educación Superior en el siglo XXI. Libro de Proceedings. Sede La Serena, Chile. 197p.

Aksela, M. and Haatainen, O. (2018). Project-based Learning (PBL) in practice: Active Teachers view of its Advantages and Challenges. In: 5th International STEM in Education Conference Proceedings: Integrated Education for the Real World, Queensland, University of Technology, Australia. 